\title{
A portable and accurate phosphate sensor using a gradient Fabry-Pérot array
}

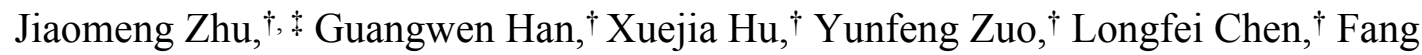
Wang, ${ }^{\dagger}$ Yi Yang, ${ }^{* \dagger}$ Fenghua Jiang, ${ }^{\S}$ Chengjun Sun, ${ }^{\S}$ Weihong Zhao, ${ }^{\Phi}$ and Xiaotian $\operatorname{Han}^{\Phi}$

$\dagger$ Key Laboratory of Artificial Micro- and Nano- Structures of Ministry of Education, School of Physics \& technology, Wuhan University, Wuhan 430072, China.

$¥$ Shenzhen Research Institute, Wuhan University, Shenzhen 518000, China.

$\S$ The First Institute of Oceanography, SOA, China

${ }^{\Phi}$ Institute of Oceanology, Chinese Academy of Sciences, China

*E-mail: yangyiys@whu.edu.cn Tel: 86-2768752989 8103 
Contents:

S-3 Reagent preparation

S-4 Figure S1. The design of the Circuit board.

S-5 Figure S1. The concentrations distribution in different channels.

S-6 Figure S2. The study of the salinity effect.

S-7 Table S1. Comparison of measuring concentration by the traditional analyzer and the smart sensor.

S-8 Table S2. The study of the light intensity. 
Reagent preparation. Potassium phosphate monobasic $\left(\mathrm{KH}_{2} \mathrm{PO}_{4}, \mathrm{AR}, 99.5 \%\right.$, Aladdin), potassium antimony tartrate $\left(\mathrm{C}_{8} \mathrm{H}_{4} \mathrm{~K}_{2} \mathrm{O}_{12} \mathrm{Sb}_{2} .3 \mathrm{H}_{2} \mathrm{O}, \mathrm{AR}, 99.5 \%\right.$, Aladdin), ammonium molybdate tetrahydrate $\left(\mathrm{H}_{24} \mathrm{Mo}_{7} \mathrm{~N}_{6} \mathrm{O}_{24} \cdot 4 \mathrm{H}_{2} \mathrm{O}, \mathrm{AR}, 99 \%\right.$, Aladdin), sodium chloride $(\mathrm{NaCl}$, $\mathrm{AR}, 99.5 \%$, Aladdin), ascorbic acid $\left(\mathrm{C}_{6} \mathrm{H}_{8} \mathrm{O}_{6}, \mathrm{AR},>99.0 \%\right.$, Aladdin), concentrated sulfuric acid (ACS reagent 37\% and DI-water, Aladdin) and deionized water (18.25 M $\Omega . c m$, DI water) were required in this research. Phosphate standard solution $(C=100 \mu \mathrm{M})$ was prepared by dissolving dried potassium dihydrogen phosphate $(0.136 \mathrm{~g})$ in $10 \mathrm{ml}$ sulfuric acid solution and adding DI water to $1000 \mathrm{ml}$ for a constant volume, then diluting it tenfold. The chromogenic agent contains two parts: chromogenic agent 1 is ascorbic acid solution $(C=100 \mu \mathrm{M})$, it can be obtained by dissolving ascorbic acid $(0.176 \mathrm{~g})$ in $1000 \mathrm{ml}$ DI water, and then diluting it tenfold. The chromogenic agent 2 is prepared by mixing ammonium molybdate solution $(C=100 \mu \mathrm{M})$ and antimony potassium tartrate solution $(C=100 \mu \mathrm{M})$ in sulfuric acid solution. 
(a)

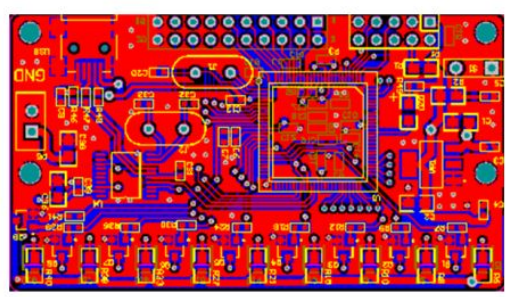

(c)

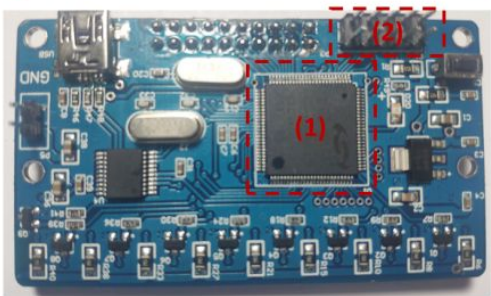

(b)

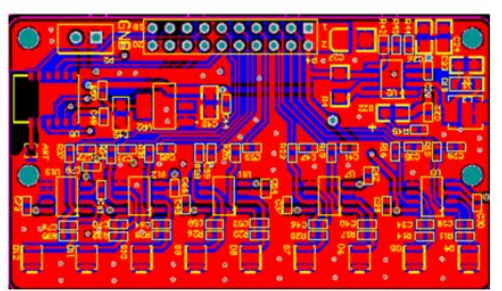

(d)

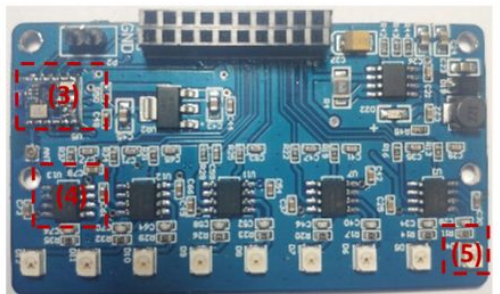

Figure S-1. (a) and (b) are the design of the PCB. (c) and (d) are images of top circuit board and bottom circuit board, where (1) is the MCU, (2) is a serial port connected to the MCU, (3) is a Bluetooth, (4) is an amplifier, and (5) is a SMD phototransistor. 


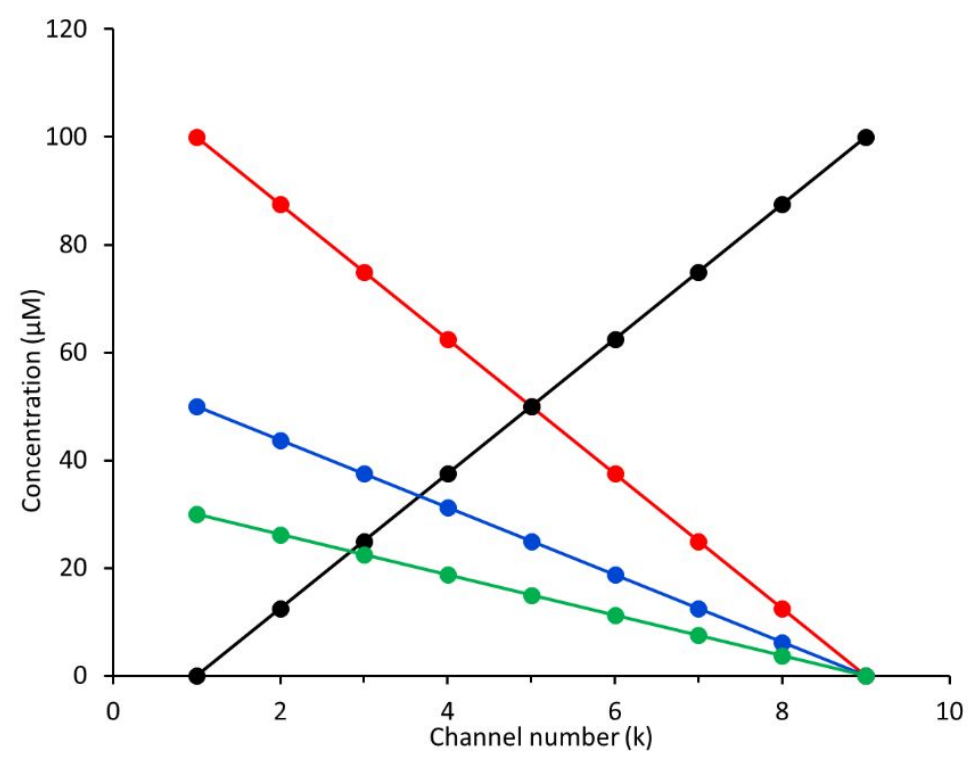

Figure S-2. The concentrations of the different channels, where the black line represents chromogenic agent $\left(C_{2}=100 \mu \mathrm{M}\right)$, other are phosphate standard solutions $\left(C_{1}=100 \mu \mathrm{M}\right.$, red line, $50 \mu \mathrm{M}$, blue line and $30 \mu \mathrm{M}$, green line). 


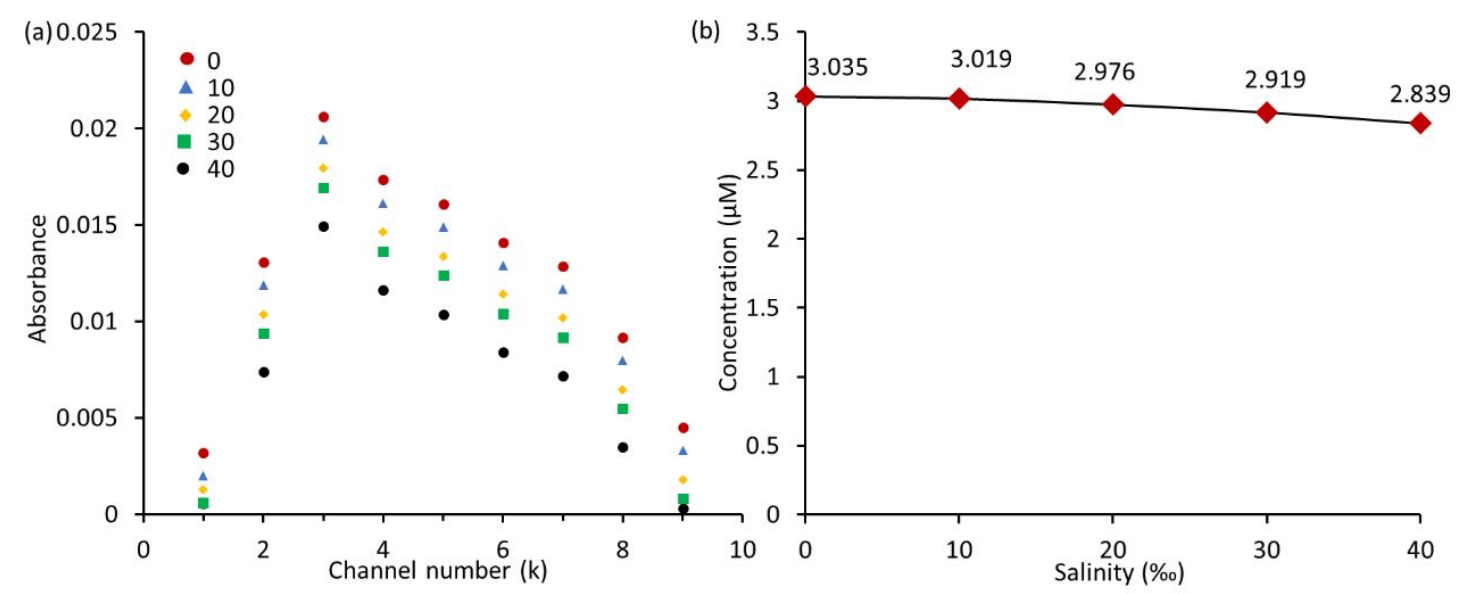

Figure S-3. (a) The absorbance of the different channels and (b) the measuring concentrations when the salinity of the phosphate standard solution is $0,10 \%, 20 \%, 30 \%$, $40 \%$ respectively. Here, the concentration of the phosphate standard solution is $3 \mu \mathrm{M}, \mathrm{n}=3$. 
Table S1. Comparison of measuring concentration by the traditional analyzer and the smart sensor. $(\mathrm{n}=3)$

\begin{tabular}{lll}
\hline Phosphate detection & Traditional analyzer $(\mu \mathrm{M})$ & Smart sensor $(\mu \mathrm{M})$ \\
\hline Sample 1 & $1.08 \pm 0.019$ & $1.09 \pm 0.016$ \\
Sample 2 & $1.36 \pm 0.043$ & $1.34 \pm 0.028$ \\
Sample 3 & $31.43 \pm 0.375$ & $31.45 \pm 0.223$ \\
\hline
\end{tabular}


Table S2. The absorbance of different channels when the light intensity was changed at 0.9 times and 1.1 times. And the concentration of phosphate sample is $100 \mu \mathrm{M}$.

\begin{tabular}{llllllllll}
\hline & 1 & 2 & 3 & 4 & 5 & 6 & 7 & 8 & 9 \\
\hline 0.9 & 0.046 & 0.189 & 0.459 & 0.615 & 0.725 & 0.612 & 0.411 & 0.177 & 0.047 \\
1 & 0.001 & 0.144 & 0.414 & 0.57 & 0.680 & 0.567 & 0.366 & 0.132 & 0.002 \\
1.1 & -0.044 & 0.099 & 0.369 & 0.525 & 0.635 & 0.522 & 0.321 & 0.087 & -0.043 \\
\hline
\end{tabular}

